\title{
ON-SKY WIDE-FIELD ADAPTIVE OPTICS CORRECTION USING MULTIPLE LASER GUIDE STARS AT THE MMT
}

\author{
Christoph Baranec ${ }^{1,3}$, Michael Hart $^{1}$, N. Mark Milton ${ }^{1}$, Thomas Stalcup ${ }^{2}$, Keith Powell $^{1}$, Miguel Snyder ${ }^{1,4}$, \\ Vidhya Vaitheeswaran ${ }^{1}$, Don McCarthy ${ }^{1}$, and Craig Kulesa ${ }^{1}$ \\ ${ }^{1}$ Steward Observatory, University of Arizona, Tucson, AZ 85721, USA; baranec@astro.caltech.edu \\ ${ }^{2}$ MMT Observatory, Tucson, AZ 85721, USA \\ Received 2008 September 1; accepted 2008 November 28; published 2009 March 10
}

\begin{abstract}
We describe results from the first astronomical adaptive optics (AO) system to use multiple laser guide stars, located at the $6.5 \mathrm{~m}$ MMT telescope in Arizona. Its initial operational mode, ground-layer adaptive optics (GLAO), provides uniform stellar wave front correction within the $2^{\prime}$ diameter laser beacon constellation, reducing the stellar image widths by as much as $53 \%$, from 0 '.70 to 0.33 at $\lambda=2.14 \mu \mathrm{m}$. GLAO is achieved by applying a correction to the telescope's adaptive secondary mirror that is an average of wave front measurements from five laser beacons supplemented with image motion from a faint stellar source. Optimization of the AO system in subsequent commissioning runs will further improve correction performance where it is predicted to deliver $0 . " 1-0 . " 2$ resolution in the near-infrared during a majority of seeing conditions.
\end{abstract}

Key words: atmospheric effects - instrumentation: adaptive optics - instrumentation: high angular resolution telescopes

Online-only material: color figure

\section{INTRODUCTION}

Until very recently, adaptive optics (AO) systems supporting astronomical observing have used single guide stars to measure atmospheric turbulence. This has limited the best optical correction to a single target, with correction degrading as a function of increasing field angle. By using multiple guide stars, many types of wide-field AO correction can be implemented. The simplest of these, ground-layer adaptive optics (GLAO), was suggested by Rigaut (2002) as a way to improve wide-field imaging for large telescopes. Wave front measurements from guide stars located far from each other ( 2 to $>10$ arcmin) can be averaged to estimate the turbulence close to the telescope aperture. A partially corrected field over the guide star constellation is produced when this low-lying turbulence is compensated. Alternatively, single Rayleigh laser guide star (LGS) systems at smaller telescopes such as the $4.2 \mathrm{~m}$ William Herschel Telescope (Martin et al. 2008) and planned for the $4.1 \mathrm{~m}$ Southern Astrophysical Research (Tokovinin et al. 2008) telescope can produce wave front compensation over fields of 1.5 and $3^{\prime}$, respectively. However, a single-beacon GLAO implementation will not be effective for larger apertures which suffer from stronger focal anisoplanatism effects that limit the accuracy of the ground-layer turbulence measurements (Andersen et al. 2006). Multiple LGSs will also be required for GLAO implementations that achieve full sky coverage on the next generation of extremely large (greater than $25 \mathrm{~m}$ ) class telescopes.

Simulations have predicted that multiple-beacon GLAO will effectively and consistently improve the atmospheric seeing (Rigaut 2002; Andersen et al. 2006; Le Louran \& Hubin 2006; Tokovinin 2004) and open-loop studies at the 6.5 m MMT and

\footnotetext{
3 Now at Caltech Optical Observatories, California Institute of Technology, Pasadena, CA 91125, USA.

4 Now at the Department of the Army.
}

$1.5 \mathrm{~m}$ Kuiper telescopes predict that GLAO can reduce wave front aberration by up to $\sim 45 \%$ (Lloyd-Hart et al. 2005; Baranec et al. 2007b, 2007c). Using simultaneous wave front measurements of five LGSs and a stellar source at the MMT, synthetic point-spread functions (PSFs) have been calculated from the residual wave front errors of open-loop ground-layer correction (Lloyd-Hart et al. 2006b; Baranec et al. 2007c). During roughly median-seeing conditions $\left(r_{0}=14.8 \mathrm{~cm}\right.$ at $\lambda=500 \mathrm{~nm}$ ), the full width at half-maximum (FWHM) of the corrected PSF was calculated to be $0{ }^{\prime \prime} 12$ and $0^{\prime \prime} 16$ in the $K$ and $H$ bands, respectively. Azimuthally averaged PSF profiles of both the uncorrected and open-loop ground-layer-corrected stellar PSFs are presented in Figure 1. During more favorable seeing conditions $\left(r_{0}=22.6 \mathrm{~cm}\right)$, the calculated FWHM size of the open-loop ground-layer-corrected PSFs were 0 '. 10 and 0 "'.14 in the $K$ and $H$ bands, respectively. Although the performance of GLAO is affected by both the changing seeing conditions and the relative strength of ground-layer turbulence, it has been found at many sites, including at the nearby Mt. Graham and Mt. Bigelow, that typically one-half to two-thirds of the atmospheric turbulence lies in this ground layer (Andersen et al. 2006; Avila et al. 2004; Egner et al. 2006; Tokovinin \& Travouillon 2006; Tokovinin et al. 2005; Baranec et al. 2007b; Velur et al. 2006; Verin et al. 2000). Further statistics on the strength of the ground layer at the MMT need to be measured; however, it is expected that the GLAO system will be capable of delivering $0 ! 1-0 ! 2$ resolution in the near-infrared during a majority of seeing conditions.

GLAO was first demonstrated on-sky in closed loop using three bright natural guide stars (NGSs) on a 1.5 diameter with the Multi-conjugate AO Demonstrator fielded at the Very Large Telescope (VLT) in early 2007 (Marchetti et al. 2007; Bouy et al. 2008). Strehl ratios from $5 \%$ to $22.5 \%$ in the $K$ band were observed within the guide star constellation during seeing of $\sim 0$.'7. However, sky coverage and the number of accessible 

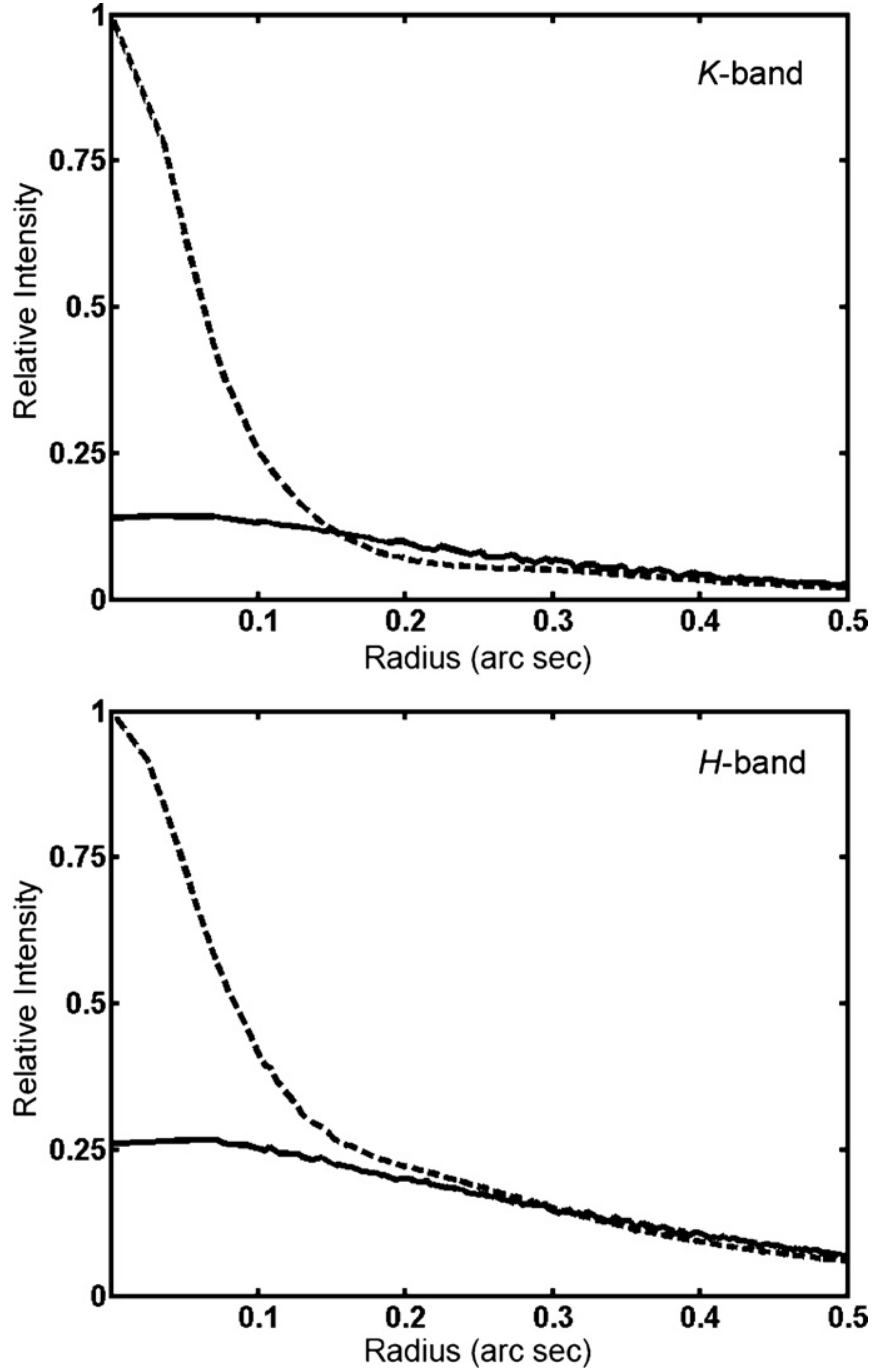

Figure 1. Radially averaged synthetic PSF profiles in $K$ and $H$ bands calculated from open-loop GLAO correction at the MMT during median seeing (LloydHart et al. 2006b). The seeing-limited PSFs are shown in solid, while the GLAO-corrected PSFs are shown as dashed lines.

science targets will be limited because there are very few suitably bright NGS constellations.

The MMT multiple laser AO system is being commissioned to support wide-field science with GLAO and narrow-field science with laser tomography AO (where Strehl is maximized at the cost of corrected field of view). In 2008 February, the system demonstrated an initial GLAO correction where stellar image widths were reduced by as much as $53 \%$, from $0 . " 70$ to $0 . " 33$ at $\lambda=2.14 \mu \mathrm{m}$. Further commissioning in 2008 May demonstrated the wide-field aberration compensation of a constellation of stars, and a reduction of the stellar wave front errors in the control space of the laser wave front sensor (WFS) by $38 \%$. The observations reported here demonstrate significant image improvement across a $110^{\prime \prime}$ field, but not yet at the anticipated resolution of $0 .^{\prime \prime} 1-0$. . $^{\prime} 2$. At the time of these observations, the GLAO system was affected by several issues in the tip-tilt loop which compromised the overall closed-loop performance. The primary problems were network delays and dropouts between the tip-tilt sensor and real-time reconstructor, responsible for unwanted random control loop delays, causing the system to be unstable at anything other than at very low control loop gain values. The tip-tilt issues have since been diagnosed and corrected; thus future observations should not be affected.

\section{EXPERIMENTAL DESIGN}

\subsection{Instrument Description}

The MMT's multiple LGS AO system comprises a laser launch telescope (Stalcup 2006), an adaptive secondary mirror (ASM; Brusa-Zappellini et al. 1998; Wildi et al. 2003), a Cassegrain mounted WFS instrument (Lloyd-Hart et al. 2005, 2006b; Baranec 2007; Baranec et al. 2007c), and a PC-based real-time reconstructor computer (Vaitheeswaran et al. 2008). The laser launch telescope, mounted above the MMT's ASM, projects a constellation of five $\lambda=532 \mathrm{~nm}$ Rayleigh LGSs on a regular $2^{\prime}$ diameter pentagon with a total on-sky power of $25 \mathrm{~W}$.

The Cassegrain WFS instrument consists of a LGS WFS, a tip-tilt sensor and a NGS WFS. The LGS WFS uses dynamically refocused optics (Georges 2003) and an electronically shuttered CCD to accumulate photon return over a range of $20-29 \mathrm{~km}$ from the telescope. Shack-Hartmann patterns for each of the five LGSs are created by a prism array (Putnam et al. 2004) with 60 subapertures in a hexapolar geometry, and captured at a rate of 400 frames per second. An electron multiplying CCD is used to obtain tip-tilt measurements from a natural star within the 2' LGS constellation at the same rate. The NGS WFS is a traditional $12 \times 12$ Shack-Hartmann sensor used for system calibration and automatic static aberration correction. The Cassegrain-mounted WFS instrument is designed to accept the current suite of MMT $f / 15$ NGS AO science instruments including PISCES (McCarthy et al. 2004), Clio (Freed et al. 2004), ARIES (McCarthy et al. 1998), and BLINC-MIRAC (Hinz et al. 2000).

The GLAO correction is calculated by reconstructor matrix multiplication in the PC-based real-time computer from 300 LGS slope pairs, 60 pairs from each of the five laser beacons, as well as a pair of slopes from the fast tip-tilt camera. The reconstructor projects each of the five sets of LGS WFS slope measurements onto an orthonormal basis of disk harmonic (DH) functions (Milton \& Lloyd-Hart 2005) in the telescope's pupil. The five LGS wave fronts are averaged by mode to produce an estimate of the ground-layer contribution to atmospheric seeing. Finally, the GLAO modal estimate is converted to actuator displacements which are transmitted to the ASM at the telescope pupil. In addition to an overall system loop gain, separate gains can be applied to the individual $\mathrm{DH}$ modes in the reconstruction for fine tuning of the system response. These are obtained from the measured modal closed-loop system response. Measured scale factors for the individual responses of the ASM actuators are also applied to account for variations in the sensitivities of the associated capacitive sensors. The DH basis functions are used instead of the traditional Zernike polynomials since they provide increased loop stability and a lower rms error for a given number of controlled modes. Zernike polynomials have large radial derivatives near the edge of the pupil, particularly for high spatial frequency modes. By contrast, the DH functions have zero radial derivatives at the edge of the pupil, placing less stress on actuators at the outer edge of the ASM and resulting in lower actuator currents and greater loop stability.

\subsection{MMT Seeing Measurements}

The results of the GLAO correction are presented in the context of seeing statistics recorded at the telescope spanning the 


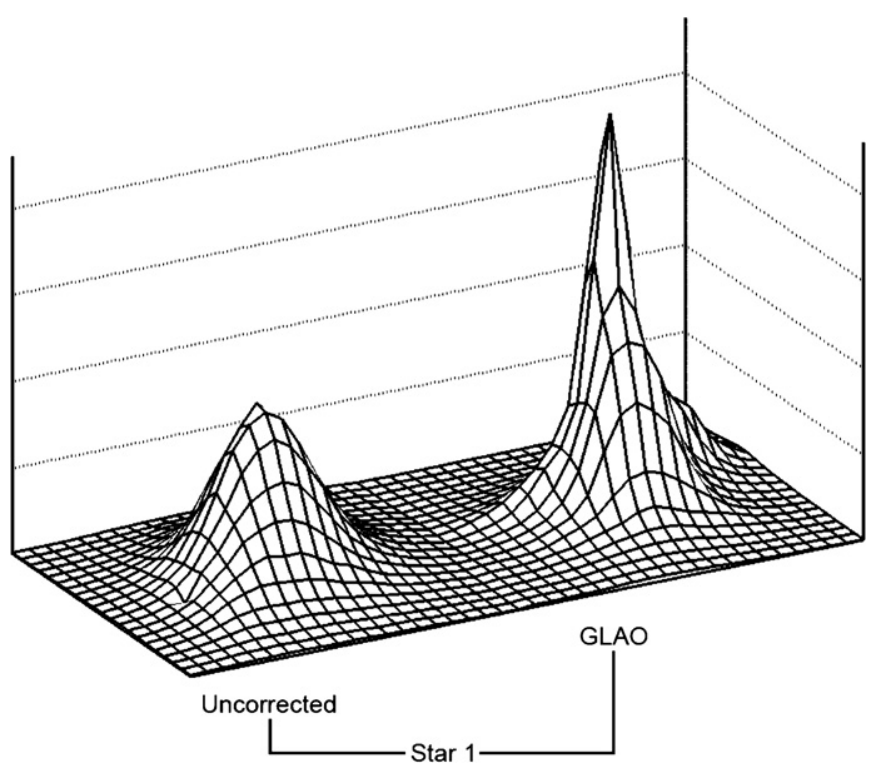

Figure 2. Comparison of PSFs for star 1 at $\lambda=2.14 \mu \mathrm{m}$. Without correction, the FWHM of the PSF is 0."70. With GLAO correction, the FWHM is $0 . " 33$. The peak intensity of the GLAO-corrected PSF is 2.3 times greater than the peak intensity of the seeing-limited PSF. Each grid point represents 107 mas.

past $5 \mathrm{yr}$. Measurements of seeing are made by calculating stellar image widths directly as observed by the Shack-Hartmann WFSs used for the active alignment of the MMT's primary mirror with its $f / 5$ and $f / 9$ secondary mirrors (Pickering et al. 2004). The sensors do not have the temporal bandwidth to resolve the dynamic atmospheric turbulence so the integrated image widths represent the best-available estimate of seeing. The WFSs currently operate at a mean wavelength of $\bar{\lambda}=$ $650 \mathrm{~nm}$ (T. Pickering 2008, private communication) and data comprise $\sim 50,000$ individual measurements taken during approximately 800 different nights. Measurements are corrected for airmass (quoted at zenith) and are extrapolated - in an overly conservative way- to infrared wavelengths by a factor of the ratio of wavelengths to the $-1 / 5$ power.

\subsection{Methods}

Data were taken to compare seeing-limited imaging with that of tip-tilt only and GLAO-corrected imaging. The images were captured with the science instrument PISCES, a near-infrared camera with a $110^{\prime \prime}$ field of view and a plate scale of 107 mas per pixel. Observations comprised consecutive $1 \mathrm{~s}$ exposures, with a rate of approximately 14 exposures per minute. During subsequent analysis, exposures were first background subtracted and flat fielded, then averaged to simulate a long exposure. Sky de-rotation was then applied post facto for images with more than one object. All images were taken with a standard $K_{s}$ or $\lambda=2.14 \mu \mathrm{m}$ narrowband filter. Image widths were calculated using the MOFFAT radial fit tool in the imexam package of IRAF.

\section{OBSERVATIONS AND ANALYSIS}

\subsection{February}

The first astronomical targets observed on the night of 2008 February 19 were a series of single stars ranging in visual magnitude from 8 to 10 , all with a declination of approximately

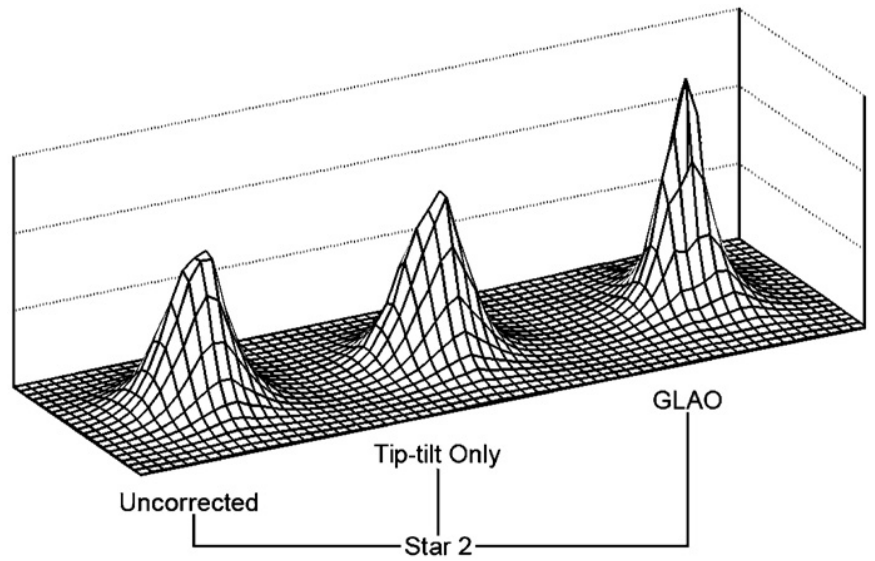

Figure 3. Comparison of PSFs for star 2 at $K_{s}$. Without correction, the FWHM of the PSF is $0 . " 60$. With tip-tilt only correction, the FWHM is 0." 56 , with an increase in peak intensity of $8 \%$. With GLAO correction, the FWHM is $0 . " 39$, with an increase of peak intensity over the seeing-limited PSF of $48 \%$. Each grid point represents 107 mas.

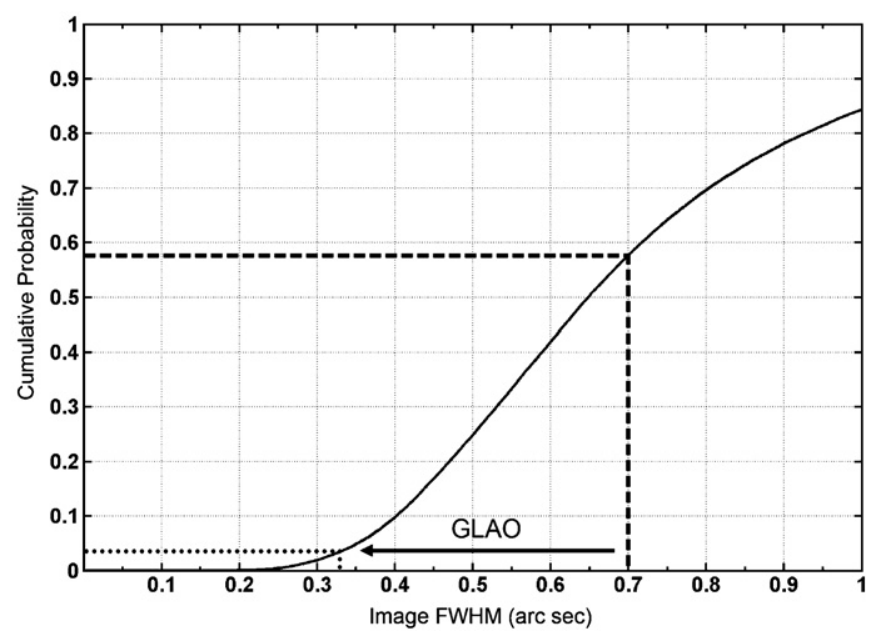

Figure 4. Conservative estimate of the MMT's cumulative seeing diagram for $\lambda=2.14 \mu \mathrm{m}$, extrapolated from measurements recorded at visible wavelengths over a 5 yr period. Observations of star 1 are indicated showing the native seeing (dashed line) and during GLAO correction (dotted line).

$+40^{\circ}$. The stars were located approximately in the center of the laser beacon constellation with tip-tilt sensing done using the target star. Figures 2 and 3 show examples of the stellar PSF during seeing-limited, tip-tilt only, and the GLAO correction at $K_{s}$ and $\lambda=2.14 \mu \mathrm{m}$. Each image is an average of approximately $601 \mathrm{~s}$ exposures.

Star 1 has a seeing-limited FWHM of $0 .{ }^{\prime \prime} 70$, while the closedloop PSF has a FWHM of $0 . " 33$, a reduction of the image width of $53 \%$, with a factor of 2.3 increase in relative peak intensity. The GLAO-corrected PSF shows some asymmetry which is similar in each of the 60 exposures used to create the mean image in Figure 2 indicating that the system was limited by uncorrected noncommon path errors. The reduction in image width with GLAO can be compared with the cumulative seeing distribution measured at the MMT as presented in Figure 4. The GLAO correction represents an effective improvement in seeing from somewhat worse than median for the site, at the 58th percentile, to excellent, at the fourth percentile. 


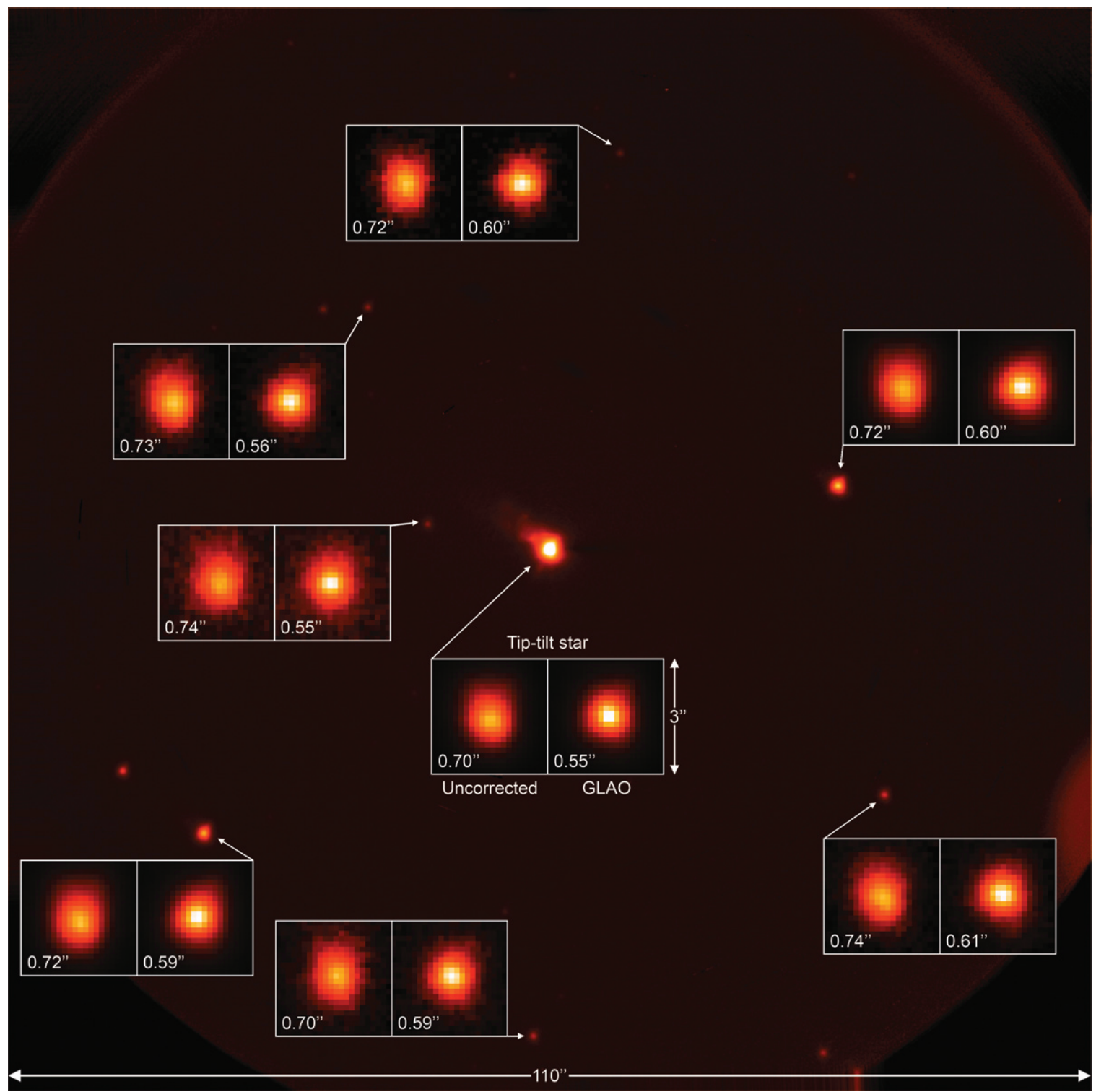

Figure 5. PISCES field showing $4 \times$ magnified images of PSFs with and without ground-layer correction. Each box is linearly scaled and normalized to the peak intensity, while the background image is logarithmically stretched to give greater visibility of faint field stars. The LGSs are located just outside this field on a $120^{\prime \prime}$ diameter pentagon.

(A color version of this figure is available in the online journal.)

During observations of star 2, the GLAO correction was modest. A $10 \%$ decrease in image width was observed with the tip-tilt only correction; however, with full ground-layer correction, the FWHM of the image is reduced by $35 \%$, from $0 . " 60$ to $0 . " 39$. This corresponds to an effective seeing improvement from the 42 nd to the 9 th percentile.

Although the results from the 2008 February telescope run demonstrate successful closed-loop GLAO operation, the level of correction did not achieve the final expected system performance of $0^{\prime \prime} 1-0^{\prime \prime} .2$ images in the $K$ band. This is attributable to three factors. Time to implement and test the system for measuring and correcting noncommon path and other static aberrations was cut short due to inclement weather. This feature was implemented and initial testing was completed in the subsequent 2008 May run. Second, and more seriously, there were random network transmission delays and dropouts in the tip-tilt control loop, which unbeknownst to us at the time, severely affected loop stability. In addition, the elevation axis servo control sys- tem of the MMT telescope was exhibiting technical problems resulting in the amplification of wind-driven oscillations at 2.25 Hz. The AO loop gain had to be significantly reduced in the presence of the latter two factors in order to maintain stable closed-loop GLAO operation, compromising the final level of image correction.

\subsection{May}

Additional observations were carried out in 2008 May in order to implement and test automated static aberration correction with the NGS WFS and to characterize the uniformity of the GLAO-corrected PSF over the 110" field. Unfortunately, these results were again severely affected by the tip-tilt network issues and continuing problems with the MMT telescope elevation axis servo control system. Automated static aberration correction for the MMT LGS WFS system is achieved by measuring the difference in the long-term average open-loop and closed- 
Table 1

Comparison of Residual Wave Front Error With and Without GLAO Compensation

\begin{tabular}{|c|c|c|c|c|c|c|c|c|c|c|c|c|c|}
\hline \multirow[t]{2}{*}{ Correction Method } & \multirow[t]{2}{*}{ Set Number } & \multicolumn{2}{|c|}{ Zernike Mode } & \multicolumn{10}{|c|}{ Zernike Order } \\
\hline & & EL-tilt & AZ-tilt & 1 & 2 & 3 & 4 & 5 & 6 & 7 & 8 & $1-8$ & $2-8$ \\
\hline \multirow{2}{*}{ Uncorrected } & 1 & $830(0.109)$ & $778(0.102)$ & 1137 & 486 & 313 & 230 & 187 & 153 & 131 & 118 & 1330 & 690 \\
\hline & 2 & $868(0.114)$ & $867(0.114)$ & 1227 & 460 & 307 & 221 & 175 & 145 & 127 & 114 & 1393 & 660 \\
\hline \multirow[t]{2}{*}{ GLAO } & 1 & $477(0.062)$ & $473(0.062)$ & 671 & 244 & 213 & 145 & 130 & 116 & 115 & 107 & 794 & 425 \\
\hline & 2 & $501(0.066)$ & $452(0.059)$ & 675 & 235 & 209 & 138 & 125 & 109 & 108 & 100 & 790 & 409 \\
\hline Mean \% Correction & & 42 & 44 & 43 & 49 & 32 & 37 & 30 & 24 & 14 & 11 & 42 & 38 \\
\hline
\end{tabular}

Notes. rms stellar wave front error in $\mathrm{nm}$, of individual modes or summed in quadrature over all the modes in the indicated Zernike orders. Parenthesized values represent the rms image position error in arcseconds. The bottom row represents the mean percentage correction with GLAO.

loop GLAO aberrations of the tip-tilt star with the NGS WFS, similar to the quasistatic calibration with the Keck LGS AO system (van Dam et al. 2006). The change in the mean NGS WFS slopes is reconstructed to produce a modal estimate of the quasistatic nonatmospheric aberrations. These modal corrections are converted to LGS WFS centroid offsets using the LGS WFS influence matrix. Finally, these offsets are used to shift the zero points of the LGS WFS slope calculation during GLAO closed-loop operation.

Data were taken of an $m_{V}=9 \operatorname{star}\left(20^{\mathrm{h}} 01^{\mathrm{m}} 16^{\mathrm{s}} .72,20^{\circ} 41^{\prime} 29^{\prime \prime} .8\right.$, J2000) with many field stars seen in the $110^{\prime \prime}$ PISCES field of view. Figure 5 shows an image of the field, with brighter stars magnified, shown both uncorrected and with ground-layer correction. The stellar images are rescaled in intensity for clarity. Both corrected and uncorrected data are averages of $701 \mathrm{~s}$ exposures with the narrowband $\lambda=2.14 \mu \mathrm{m}$ filter. Tip-tilt signals were obtained from the bright star in the center of the field, which was also used to sense static aberrations. Image improvement was again modest: the tip-tilt star has a measured FWHM of 0." 70 in open loop and 0." 55 in GLAO closed loop, a decrease of only $21 \%$. Over the entire field, both the uncorrected and GLAO corrected PSFs are fairly constant, with a mean seeing-limited FWHM of 0".72 \pm 0 0.01 for the brightest 12 stars in this field and a mean GLAO-corrected FWHM of 0'.58 \pm 0.03 for the same stars. In contrast to the results from the February run in Figure 2, the corrected PSFs are round, showing the effect of removing the static aberration by reference to the NGS WFS. We expect a substantial overall improvement in the PSF FWHM during future observations now that the vibration issues have been resolved and we are able to increase the GLAO loop gain, however, a decrease in closed-loop PSF uniformity may be seen due to anisoplanatic effects.

During these observations, the NGS WFS was passively collecting data in both open- and closed-loop operations of the tip-tilt star at 180 frames per second. These data have been analyzed to evaluate the closed-loop performance in a manner independent of static aberrations. Table 1 shows the rms modal amplitudes reconstructed from the NGS WFS measurements, grouped by radial order. We show the more familiar Zernike modes rather than the $\mathrm{DH}$ modes used in the real-time control. GLAO-corrected $42 \%$ of the measured total rms wave front error, with a reduction in the modes controlled by the LGS WFS (orders 2-8) of 38\%, consistent with previous open-loop studies of GLAO performance at the MMT (Baranec et al. 2007c). However, the reduction of $43 \%$ for the tip-tilt modes is much lower than the greater than $80 \%$ correction obtained in previous analyses and attributable to the low tip-tilt loop gains and the servo oscillations experienced during this run. Note that even though there was a resonance in the telescope's elevation drive, the total power in the orthogonal tilt modes were quite similar during both open- and closed-loop observations, differing by at most $11 \%$, and as a consequence would not have affected an asymmetry in the stellar PSFs.

\subsection{Control System Analysis}

There were two different control loops used in the closedloop GLAO system: one for the NGS tip-tilt sensor and the other for the high-order LGS WFS. Both loops used an integral control law and had a sample rate of $400 \mathrm{~Hz}$. The tip-tilt loop was unfortunately hindered by two technical issues. It was discovered during an examination of telemetry data that there were randomly varying network delays and dropouts in the transmission of the tip-tilt data between the tip-tilt sensor and real-time wave front reconstruction computer. This would cause the tip-tilt control loop to act erratically and not follow the atmospheric tip-tilt signal. In addition, the $2.25 \mathrm{~Hz}$ tilt signal produced by the oscillating elevation drive increased the required actuator stroke at the edges of the ASM. With the combination of these effects, the ASM would quickly hit its safety limits for voice coil actuator current at the edges, breaking the loop-for even modest gains in the tip-tilt loop. To maintain closed-loop operation for any appreciable amount of time, the gain was severely reduced, compromising correction. Figure 6 shows the open- and closed-loop power spectra for selected Zernike modes as measured by the NGS WFS. The power spectra show that despite low gain the GLAO control is removing a significant portion of the aberrations at very low frequencies, below $\sim 1 \mathrm{~Hz}$, for the tip-tilt modes, and even a $46 \%$ reduction in the $2.25 \mathrm{~Hz}$ oscillation in elevation. The random network transmission delays, however, created a large overshoot in higher frequencies, hindering the ability to recover good image quality.

The disturbance-rejection frequency response of highorder loop, which was not affected by the tip-tilt issues, was modeled at different gain values (Figure 7). At a gain of 0.15 , the disturbance rejection bandwidth is $\sim 20 \mathrm{~Hz}$ with a low-frequency slope of $20 \mathrm{~dB} /$ decade. To maintain the closed-loop operation of the AO system during observations, the overall system gain was reduced to approximately 0.015 . At this gain, the bandwidth is limited, in the range of $3-5 \mathrm{~Hz}$. The power spectra of higher-order modes (e.g., for the astigmatism modes shown in Figure 6) show correction out to a higher bandwidth of $\sim 2.5 \mathrm{~Hz}$ due to the limited influence from the elevation servo resonance. While it is possible that the bandwidth of the higher-order modes could have been improved 

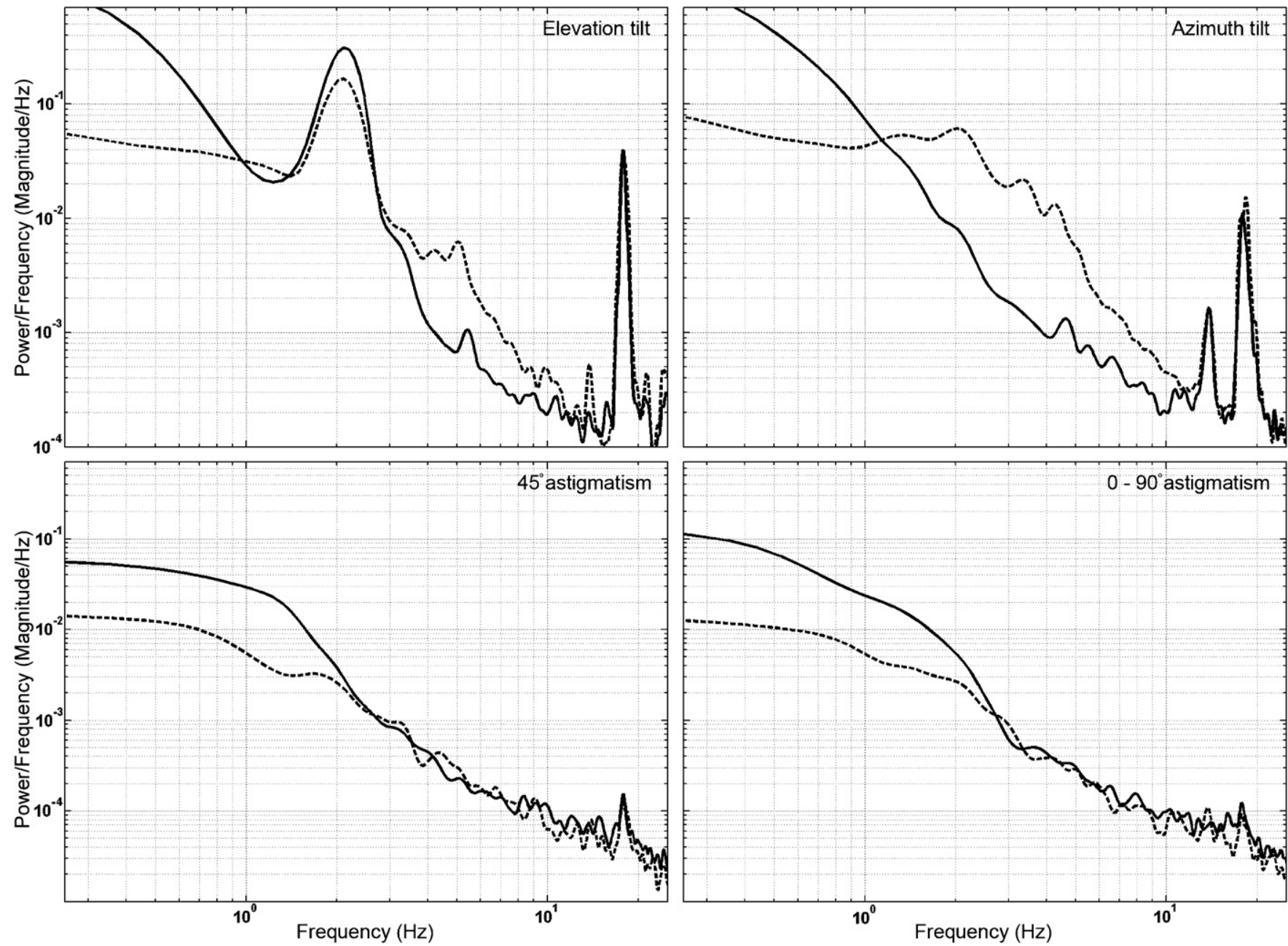

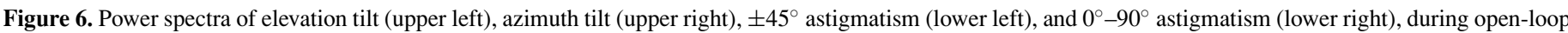

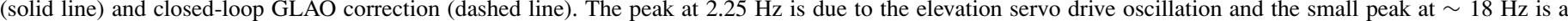
vibrational mode of the secondary hub.

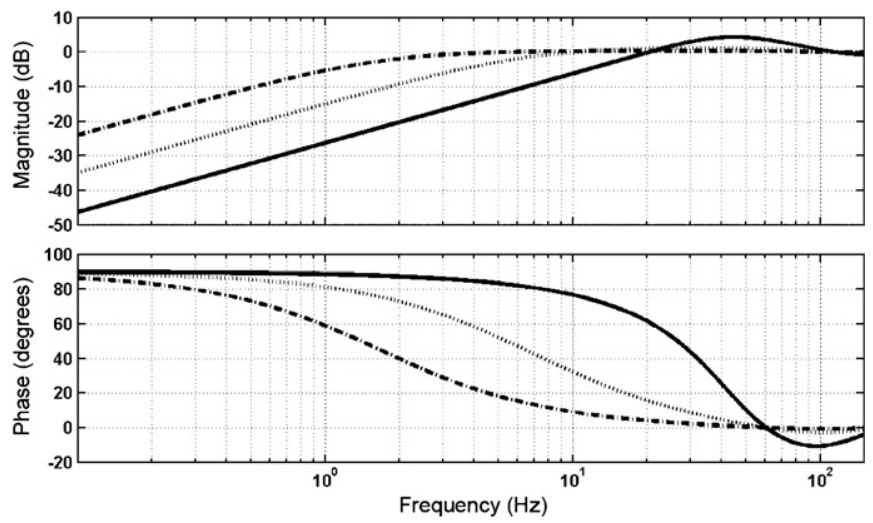

Figure 7. Modeled disturbance rejection frequency response of the high-order modes in the GLAO control system with a sample rate of $400 \mathrm{~Hz}$. System gains of 0.15 (solid line), 0.05 (dotted line), and 0.015 (dot-dashed line) are shown.

by taking advantage of the mode-dependent gains, a conservative approach to controlling the ASM was adopted, and only the overall system gain was adjusted to achieve loop stability. With the elevation axis servo oscillation issue now resolved, we expect the closed-loop bandwidth during future observations to increase to $\sim 20 \mathrm{~Hz}$ once the loop gain is increased to 0.15 and the modal gains are fine tuned. We intend to also implement a more sophisticated proportional-integral-derivative controller which should further increase the bandwidth of the controller to $\sim 30 \mathrm{~Hz}$, significantly reducing the residual wave front error.

\section{CONCLUSIONS}

The multiple LGS AO program at the MMT is exploring the practical techniques required for wave front sensing for groundlayer and tomographic correction. Closed-loop GLAO operation was successfully demonstrated in 2008 February where image correction reduced stellar image widths by as much as $53 \%$ at $\lambda=2.14 \mu \mathrm{m}$. Wide-field, $\sim 2^{\prime}$ diameter, correction was demonstrated in 2008 May upon successful testing of the static aberration correction and additional system characterization. Optimization of the system in subsequent commissioning runs in the fall of 2008 will further improve correction performance and is expected to deliver $0.1-0^{\prime \prime} .2$ resolution during a majority of seeing conditions. Additional narrow-field PSF characterization will be done in the thermal infrared $(3.5-4.8 \mu \mathrm{m})$ where Strehl ratios of $30-40 \%$ are expected using Clio with a plate scale of 48 mas per pixel. Imaging with the Clio and PISCES cameras will allow for the exploration of parameters which affect the ground-layer AO correction. In particular, variables such as control gain, reconstruction basis set, and the number of controlled modes, will be optimized. The effect of observing 
conditions, such as the brightness and field location of the tilt star, will also be explored to support science programs by anticipating the improved resolution and sensitivity of the ground-layer AO correction.

Early shared risk scientific programs will focus on seeing improvement with GLAO, taking advantage of the existing near-infrared instrumentation (Lloyd-Hart et al. 2006c), with plans for developing further capability over larger fields of view (Baranec et al. 2007a). The exploitation of routine near-infrared seeing of $0^{\prime} .2$ or better over a field of several arcminutes is likely to be very productive, both for imaging and high-resolution multi-object spectroscopy where the manifold improvement in encircled energy within $0^{\prime \prime} .2$ will be of particular value.

Development of the MMT laser AO system will lead to a greater understanding of the important factors in designing and operating future multiple guide star AO systems such as the ones being planned for the $8.1 \mathrm{~m}$ Gemini North (Szeto et al. 2006) and Gemini South (Boccas et al. 2008) telescopes, the $8.2 \mathrm{~m}$ VLT (Hubin et al. 2005; Casali et al. 2006), the $2 \times 8.4 \mathrm{~m}$ Large Binocular Telescope (Lloyd-Hart et al. 2007; Ragazzoni et al. 2003), the $10 \mathrm{~m} \mathrm{Keck}$ (Wizinowich et al. 2008; Gavel et al. 2008) telescopes, the $25.4 \mathrm{~m}$ Giant Magellan Telescope (LloydHart et al. 2006a), $30 \mathrm{~m}$ Thirty Meter Telescope (Ellerbroek et al. 2006), and $42 \mathrm{~m}$ European Extremely Large Telescope (Gilmozzi \& Spyromilio 2007).

Observations reported here were made at the MMT, a joint facility of the University of Arizona and the Smithsonian Institution. We are grateful for the continued support of the MMT Observatory staff, particularly M. Alegria, A. Milone, and J. McAfee. We thank T. Pickering for providing the MMT seeing data. This work has been supported by the National Science Foundation under grants AST-0138347 and AST-0505369 and by the University of Arizona Technology and Research Initiative Fund.

Facilities: MMT (LGS, PISCES).

\section{REFERENCES}

Andersen, D., et al. 2006, PASP, 118, 1574

Avila, R., et al. 2004, PASP, 116, 682

Baranec, C. 2007, Ph.D. thesis, Univ. Arizona

Baranec, C., Lloyd-Hart, M., \& Meyer, M. 2007a, in Proc. SPIE 6691 Astronomical Adaptive Optics Systems and Applications III, ed. R. Tyson \& M. Lloyd-Hart (San Diego: SPIE), 669106-1

Baranec, C., Lloyd-Hart, M., \& Milton, N. M. 2007b, ApJ, 661, 1332

Baranec, C., Lloyd-Hart, M., Milton, N. M., Stalcup, T., Snyder, M., Vaitheeswaran, V., McCarthy, D., \& Angel, R. 2007c, Proc. SPIE 6691, 66910
Boccas, M., et al., 2008, Proc. SPIE 7015, 70150X-1

Bouy, H., Kolb, J., Marchetti, E., Martín, E. L., Huélamo, N., \& Barrado y Navascués, D. 2008, A\&A, 477, 681

Brusa-Zappellini, G., et al. 1998, Proc. SPIE 3353, 764

Casali, M., et al. 2006, Proc. SPIE 6269, 62690W-1

Egner, S., et al. 2006, Proc. SPIE 6272, 627257-1

Ellerbroek, B., et al. 2006, Proc. SPIE 6272, 62720D-1

Freed, M., Hinz, P., Meyer, M., Milton, N. M., \& Lloyd-Hart, M. 2004, Proc. SPIE 5492, 1561

Gavel, D., et al. 2008, Proc. SPIE 7015, 701567-1

Georges, J. 2003, PhD thesis, Univ. Arizona

Gilmozzi, R., \& Spyromilio, J. 2007, ESO Messenger, 127, 11

Hinz, P., Angel, R., Woolf, N., Hoffmann, B., \& McCarthy, D. 2000, Proc. SPIE 4006, 349

Hubin, N., Arsenault, R., Conzelmann, R., Delabre, B., Le Louarn, M. Stroebele, S., \& Stuik, R. 2005, C. R. Phys., 6, 1099

Le Louarn, M., \& Hubin, N. 2006, MNRAS, 365, 1324

Lloyd-Hart, M., Angel, R., \& Green, R. 2007, Proc. SPIE 6691, 66910O-1

Lloyd-Hart, M., Angel, R., Milton, N. M., Rademacher, M., \& Codona, J. 2006a, Proc. SPIE 6272, 62720E-1

Lloyd-Hart, M., Baranec, C., Milton, N. M., Stalcup, T., Snyder, M., \& Angel, R. 2006b, Opt. Express, 14, 7541

Lloyd-Hart, M., Baranec, C., Milton, N. M., Stalcup, T., Snyder, M., Putnam, N., \& Angel, R. 2005, ApJ, 634, 679

Lloyd-Hart, M., Stalcup, T., Baranec, C., Milton, N. M., Rademacher, M., Snyder, M., Meyer, M., \& Eisenstein, D. 2006c, Proc. SPIE 6272, 62724J-1

Marchetti, E., et al. 2007, The Messenger, 129, 8

Martin, O., et al. 2008, Proc. SPIE 7015, 70154N-1

McCarthy, D., Burge, J., Angel, R., Ge, J., Sarlot, R., Fitz-Patrick, B., \& Hinz, J. 1998, Proc. SPIE 3354, 750

McCarthy, D., Ge, J., Hinz, J., Finn, R., \& de Jong, R. 2001, PASP, 113, 353

Milton, N. M., \& Lloyd-Hart, M. 2005, in OSA Conf., Disk Harmonic Functions for Adaptive Optics Simulations, http://www.opticsinfobase.org/abstract.cfm? $\mathrm{URI}=\mathrm{AO}-2005$-AWA3

Pickering, T., West, S., \& Fabricant, D. 2004, Proc. SPIE 5489, 1041

Putnam, N., Snyder, M., Stalcup, T., \& Angel, R. 2004, Proc. SPIE 5490, 1138

Ragazzoni, R., et al. 2003, Proc. SPIE 4839, 536

Rigaut, F. 2002, in Proc. ESO, Beyond Conventional Adaptive Optics, ed. E. Vernet, R. Ragazzoni, S. Esposito, \& N. Hubin (Garching), 11

Stalcup, T. 2006, Ph.D. thesis, Univ. Arizona

Stuik, R., Bacon, R., Conzelmann, R., Delabre, B, Fedrigo, E., Hubin, N., Le Louarn, M., \& Strobele, S. 2006, New Astron. Rev., 49, 618

Szeto, K., et al., 2006, Proc. SPIE 6269, 626958-1

Tokovinin, A. 2004, PASP, 116, 94

Tokovinin, A., \& Travouillon, T. 2006, MNRAS, 365, 1235

Tokovinin, A., Tighe, R., Schurter, P., Cantarutti, R., van der Bliek, N., Martinez, M., Mondaca, E., \& Montane, A. 2008, Proc. SPIE 7015, 70154C-1

Tokovinin, A., et al. 2005, PASP, 117, 395

Vaitheeswaran, V., Hinz, P., Brusa, G., Miller, D., \& Stalcup, T. 2008, Proc. SPIE 7019, 70190E-1

van Dam, M., et al. 2006, PASP, 118, 1574

Velur, V., et al. 2006, Proc. SPIE 6272, 62725C-1

Verin, J., et al. 2000, Gemini RPT-AO-G0094 (Hilo: Gemini Obs.) http://www. gemini.edu/documentation/webdocs/rpt/rpt-ao-g0094-1.ps

Wildi, F., Brusa, G., Lloyd-Hart, M., Close, L., \& Riccardi, A. 2003, Proc. SPIE 5169,17

Wizinowich, P., et al. 2008, Proc. SPIE 7015, 701511-1 